\title{
Deducing the Relation Between Viscosity and Oil-Induced Structural Changes of Viscoelastic Surfactants Using a Kinetic Approach
}

Lionel T. Fogang ${ }^{a^{*}}$, Theis I. Solling ${ }^{\mathrm{b}}$, Jan S. Pedersen ${ }^{\mathrm{c}}$, Muhammad S. Kamal ${ }^{\mathrm{d}}$, Abdullah S. Sultan ${ }^{\mathrm{e}}$

${ }^{a}$ Center for Integrative Petroleum Research, King Fahd University of Petroleum \& Minerals, Dhahran 31261, Saudi Arabia. Email: lionelfogang@kfupm.edu.sa

${ }^{\mathrm{b}}$ Center for Integrative Petroleum Research, King Fahd University of Petroleum \& Minerals, Dhahran 31261, Saudi Arabia. Email: theis.solling@kfupm.edu.sa

'Department of Chemistry and Interdisciplinary Nanoscience Center (iNANO), Aarhus University, Gustav Wieds Vej 14, Aarhus, Denmark. Email: jsp@chem.au.dk

${ }^{\mathrm{d}}$ Center for Integrative Petroleum Research, King Fahd University of Petroleum \& Minerals, Dhahran 31261, Saudi Arabia. Email: shahzadmalik@kfupm.edu.sa

eDepartment of Petroleum Engineering, King Fahd University of Petroleum \& Minerals, Dhahran 31261, Saudi Arabia. Email: sultanas@kfupm.edu.sa

*Corresponding Author: Lionel T. Fogang, Center for Integrative Petroleum Research, King Fahd University of Petroleum \& Minerals, Dhahran 31261, Saudi Arabia. Email:

lionelfogang@kfupm.edu.sa; Phone +966-13-860-8218 


\section{Methodology}

Homogenization. The placement of the homogenizer shaft into the sample container is shown in Figure S1. The hole found on the homogenizer shaft was put in contact with the oil layer to ensure complete homogenization.

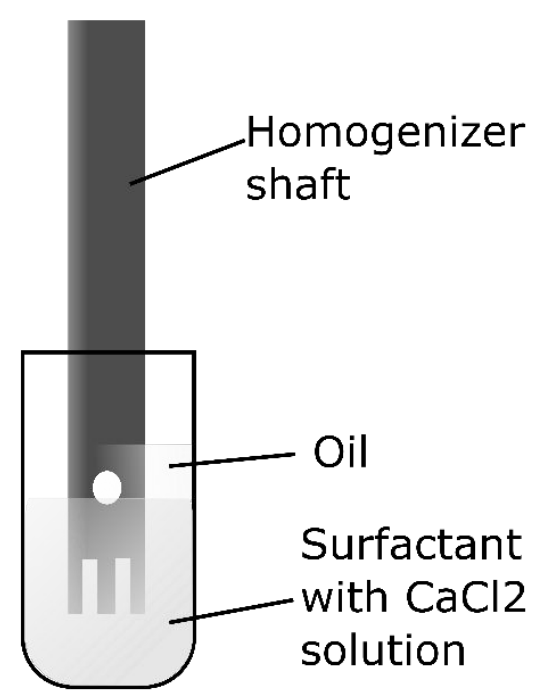

Figure S1. Placement of the homogenizer shaft into the sample container.

Choice of rheological method. A previous study used oscillatory rheology to delineate the changes in viscosity of the VES/oil mixture ${ }^{1}$. However, oscillatory rheology recorded a maximum complex viscosity of around $500 \mathrm{mPa} . \mathrm{s}$, whereas steady shear rheology could record a maximum viscosity of around 300 Pa.s. The difference in recorded viscosities arises from the shearing method and shear rate used for the measurements. The previous study used constant oscillatory shear at $10 \mathrm{rad} . \mathrm{s}^{-1}$, whereas the current study used rotational shear at $0.01 \mathrm{~s}^{-1}$.

Recording viscosity data at low shear rates is preferable as they better represent the state of the VES solution in unsheared conditions. A drop from 300 Pa.s to $5 \mathrm{mPa}$.s gives a more accurate representation of how the WLMs change into spherical micelles in non-sheared conditions unlike a drop from $500 \mathrm{mPa}$.s to $5 \mathrm{mPa}$.s. It is expected that WLMs formed by olea-tailed and erucyltailed surfactants will have such high viscosities at such low shear rates ${ }^{2-5}$.

Low oscillatory shear rates such as $0.01 \mathrm{rad} . \mathrm{s}^{-1}$ could not be used as a data point is collected approximately every $11 \mathrm{~min}$. Structural changes could occur during data collection, and thus one might miss rheological changes. Thus, it was preferred to use rotational shear as 1 min was required to obtain a data point.

Steady-shear rheology. The apparent viscosity was measured while the temperature rose to the set point. As shown in Figure S2, the temperature reached the set point after 13 minutes. The reason for not pouring the freshly homogenized sample into the concentric cylinder at the set temperature is related to the initial cooling effect. The rheometer heating device would initially cool down and then rise after the sample was poured into the concentric cylinder. That was 
because heat is been taken by the sample. Subsequently, the heating device had to raise the temperature to the set point. Structural changes would still occur due to the rapid absorption of heat during that period. Given this initial cool down, it was preferable to observe how the viscosity changes as the temperature rises as it is more controllable.

For the samples with oil, three shear rates were selected to make sure the rheometer did not obtain data below its torque sensitivity. The shear rate was set to change from $0.01 \mathrm{~s}^{-1}$ to $1 \mathrm{~s}^{-1}$ when the viscosity was below 2 Pa.s (Figure S3), and then to change from $1 \mathrm{~s}^{-1}$ to $10 \mathrm{~s}^{-1}$ when the viscosity was below $200 \mathrm{mPa}$.s. Choosing these shear rates was also based on the fact that there was a high likelihood that the flow regime of the sample will be Newtonian as shown in the previous data ${ }^{6}$. Obtaining data from the Newtonian region ensured that the WLMs were not stretched out due to the shearing from the bob ${ }^{7}$. Thus, the protocol closely replicates the effect of the oils on the VES solution without the influence of shearing.

The bob was made to rotate for $60 \mathrm{~s}$ at $0.01 \mathrm{~s}^{-1}$, and for 10 seconds at $1 \mathrm{~s}^{-1}$ and $10 \mathrm{~s}^{-1}$ before taking a datum, with an example shown in Figure S4. It is recommended that the time to shear a gel should be at least the reciprocal of the shear rate ${ }^{8}$. However, the recommendation was not followed for the equilibrated gelled VES samples at a shear rate of $0.01 \mathrm{~s}^{-1}$. That is because the viscosity of the sample increased with time and then decreased, getting close to the initial measured viscosity (see Figure S5).

The increase followed by the decrease in measured viscosity was also the reason why the samples were left unsheared before taking a datum point: The samples with $n$-decane and $n$ dodecane were left unsheared for $5 \mathrm{~min}$ before taking the next datum point because the samples were undergoing transitions which likely erased the previous shear history during the rest period. Moreover, continuous shearing was avoided to prevent the rheometer from inducing further mixing of the WLM with the oil by the stirring.

The apparent viscosity datum was the average of the last $10 \%$ of the data obtained during the rotation time as shown in Figure S6. Taking the average of the last $10 \%$ of the data accounts for the transient nature of the viscosity ${ }^{9}$. 


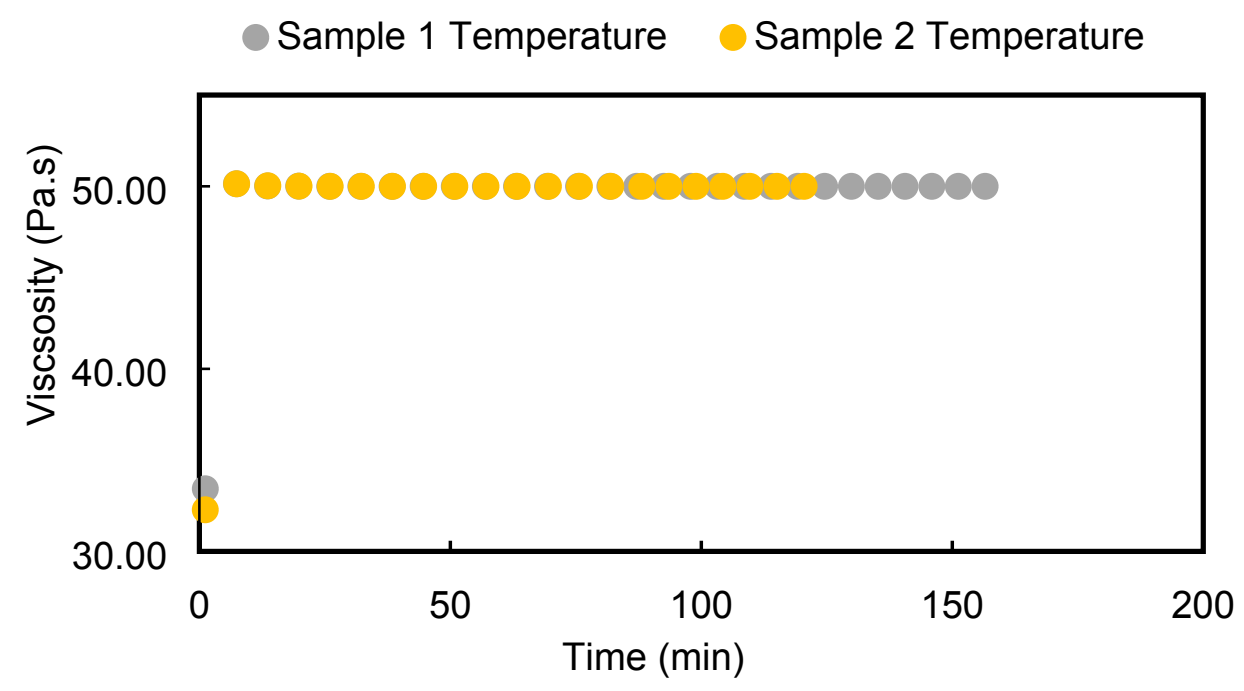

Figure S2. Temperature profile for two samples of VES/140 mM $n$-decane.

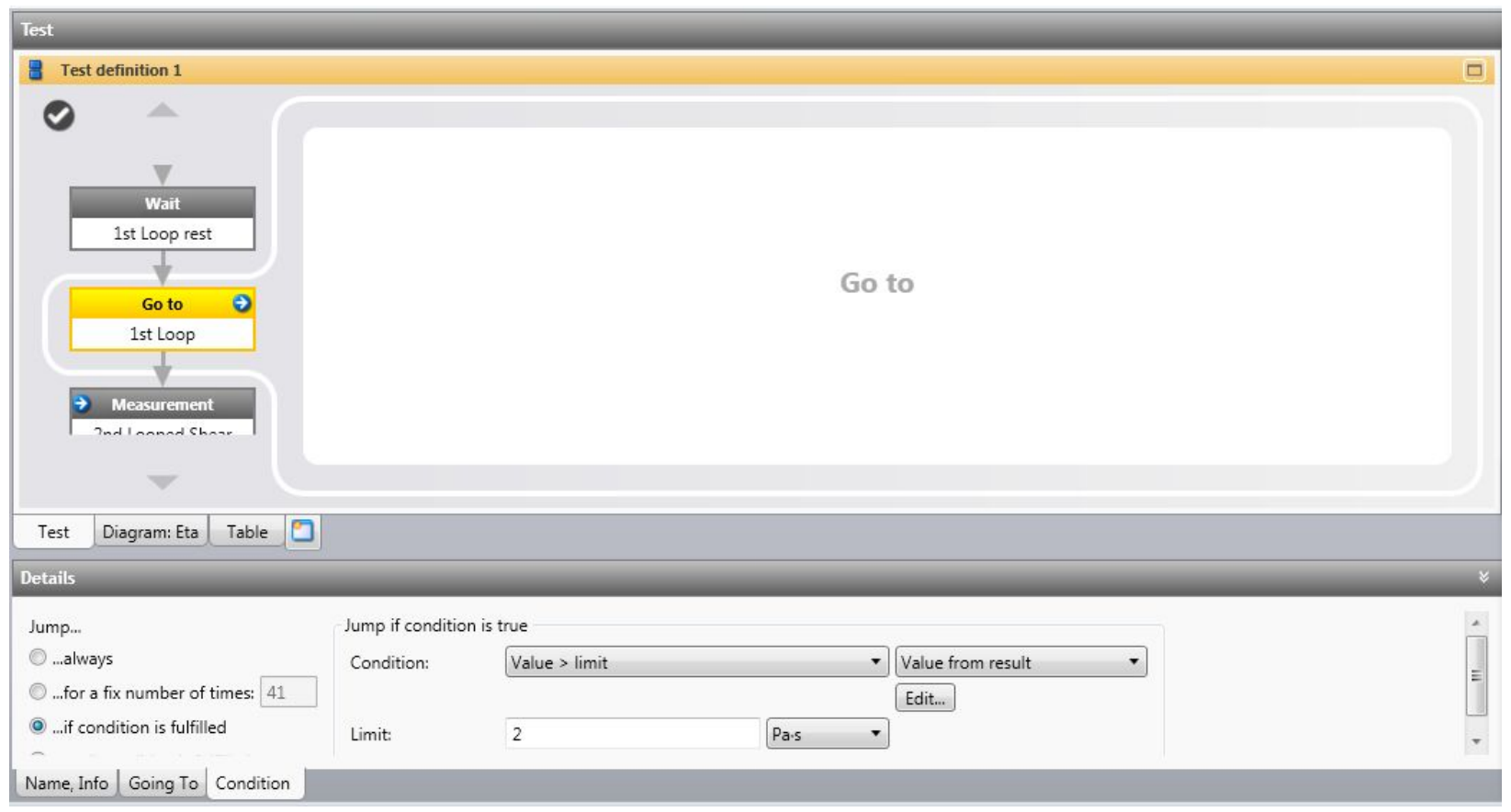

Figure S3. The first loop immediately after the first datum point is collected 


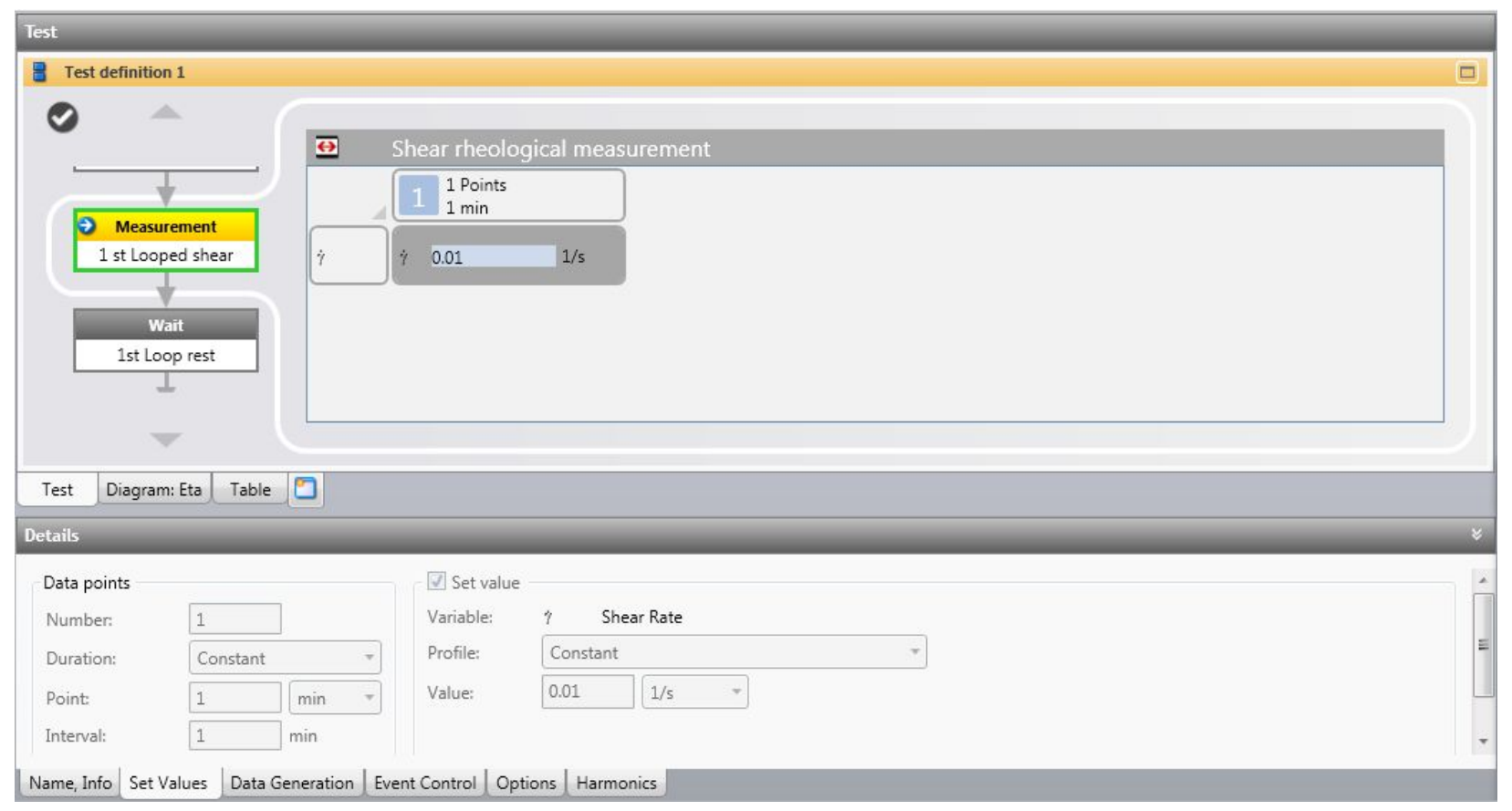

Figure S4. Setting the values for the rheological measurements.

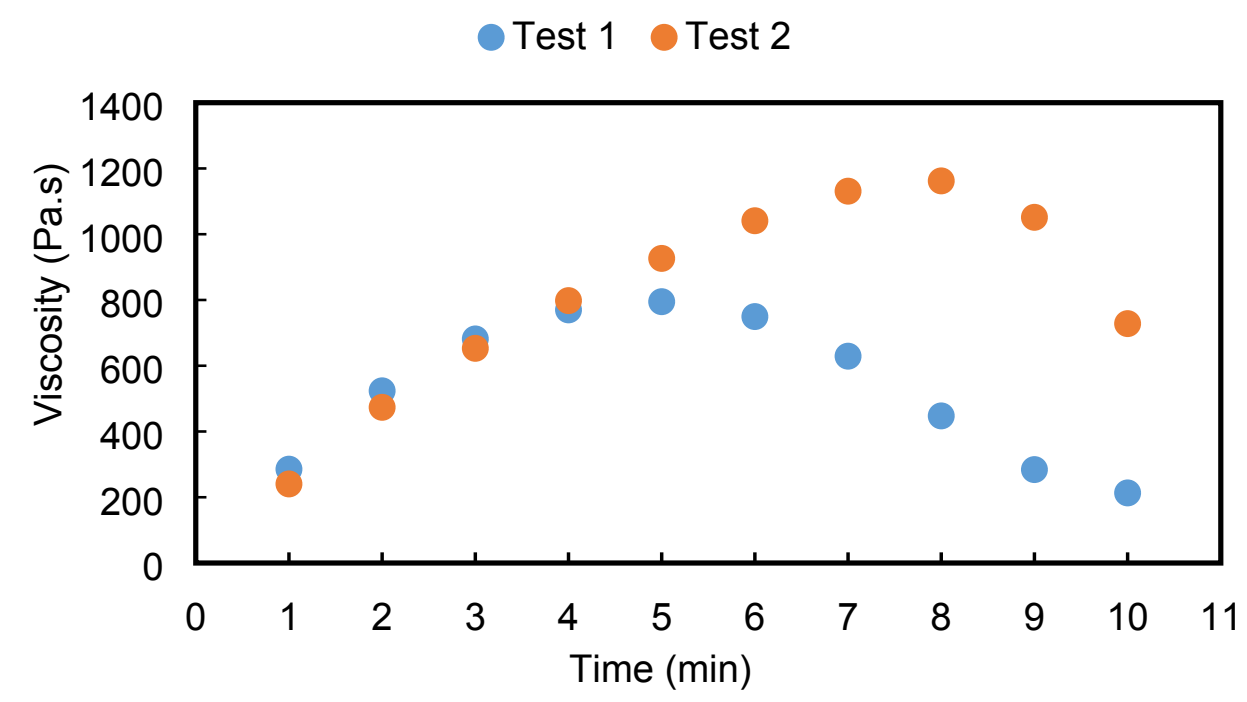

Figure S5. Change in viscosity with time of $40 \mathrm{~g} / \mathrm{L}$ surfactant at shear rate of $0.01 \mathrm{~s}^{-1}$. 


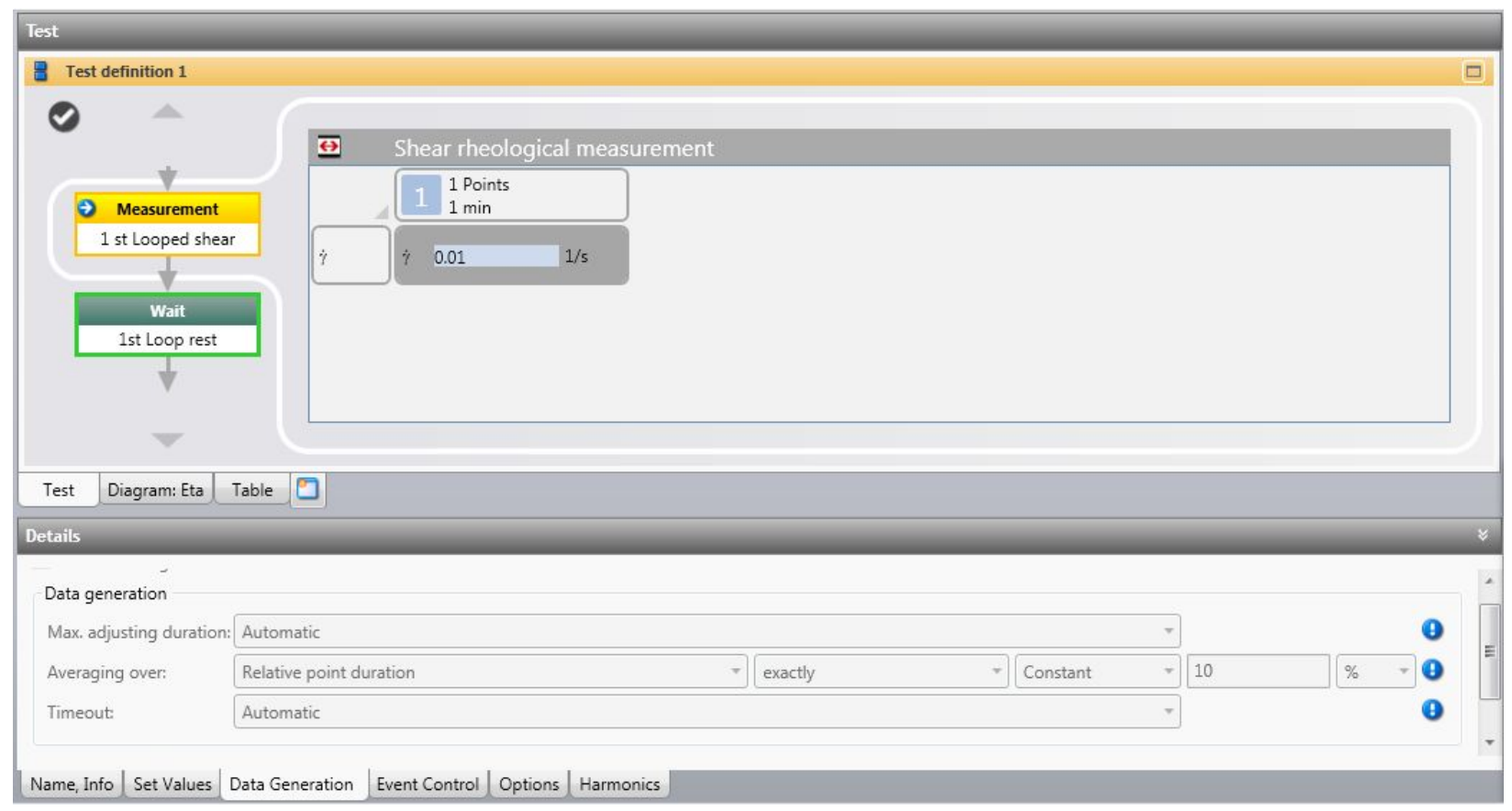

Figure S6. Apparent viscosity data generation. Averaging over a relative point duration averages a set percentage of the data collected during the rotation period. In this case, it was $10 \%$.

\section{Results}

Reproducibility Assessment. Figures 2a and 2d show the average apparent viscosity of the VES solution without oil and the coefficient of variation, respectively. The coefficient of variation increased and then decreased before it plateaued at around $2 \%$. The time at which the variation plateaued coincided with the time at which the viscosity did not change. The plateau viscosity was considered the maximum viscosity of the VES solution without oil.

Figures $2 \mathrm{~b}$ and $2 \mathrm{c}$ show how the apparent viscosities of the dummy VES solutions with $280 \mathrm{mM}$ $n$-decane change with time after controlling for both the volumes and masses of the components, respectively. Meanwhile, Figures $2 \mathrm{e}$ and $2 \mathrm{f}$ show how the coefficient of variation changes with time when controlling for both the volumes and masses of the components, respectively. The coefficient of variation increases with time up to a maximum and then decreases, whether mass or volume was controlled. The coefficient of variation reached around $90 \%$ as shown in Figure $2 \mathrm{f}$. The coefficient of variation for the controlled volume experiment was not obtainable at around $0.8 \mathrm{~h}$, which can be seen in Figure 2e. This was because the viscosity measured around this time was negative, showing that the viscosity of the sample was not measurable at that time at the set shear rate. Nevertheless, the values of the coefficient of variation show that the variability was high from the rheological results. In contrast, the SAXS results had minimal variations when compared frame by frame ${ }^{10}$. Thus, the high deviations from the viscometric data were not due to the mixing procedures, nor from the reliance on controlling volumes to prepare the solutions. With this information, the samples for the rheological experiments were prepared by controlling the volumes of each component of the mixture. 
(a)

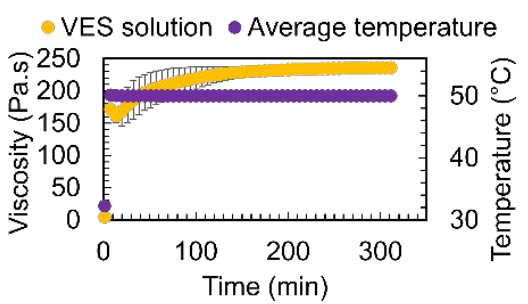

(d)

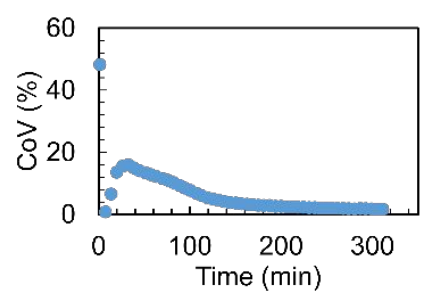

(b)

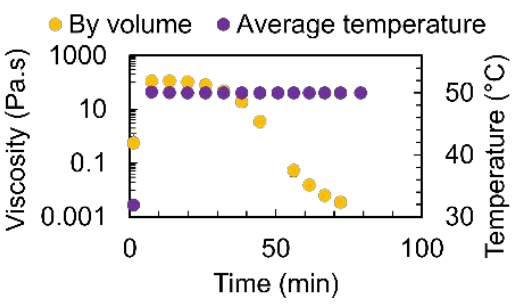

(e)

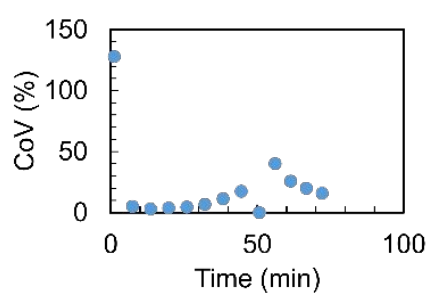

(c)

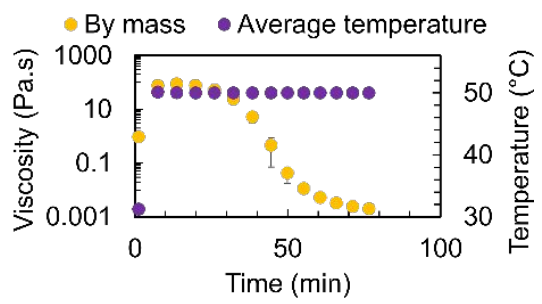

(f)

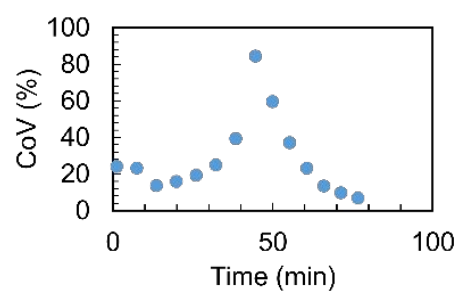

Figure S7. Average apparent time-dependent viscosity (three experiments) of VES solution without and with $280 \mathrm{mM} n$-decane (top row) along with the coefficient of variations (CoV, bottom row). The results from left to right are for the $40 \mathrm{~g} / \mathrm{L} \mathrm{VES} \mathrm{solution,} \mathrm{controlled} \mathrm{volume} \mathrm{of}$ the dummy VES solution, and controlled mass of the dummy VES solution, respectively.

Table S1. Straight line equations describing the contributions of each particle to the overall scattering, where $t$ is time in minutes.

\begin{tabular}{lll}
\hline $\begin{array}{l}\text { n-decane } \\
\text { concentration }\end{array}$ & Cylindrical particles & Spherical particles \\
\hline $280 \mathrm{mM}$ & Scale (atomic units $)=-2.87 \times 10^{-3} \mathrm{t}+$ & Scale (atomic units $)=3.96 \times 10^{-3} \mathrm{t}-$ \\
& $3.01 \times 10^{-1}\left(\mathrm{R}^{2}=0.991\right)$ & $5.09 \times 10^{-2}\left(\mathrm{R}^{2}=0.983\right)$ \\
& & \\
$140 \mathrm{mM}$ & Scale (atomic units $)=-9.89 \times 10^{-4} \mathrm{t}+$ & Scale (atomic units $)=2.03 \times 10^{-03} \mathrm{t}-$ \\
& $2.83 \times 10^{-1}\left(\mathrm{R}^{2}=0.985\right)$ & $1.49 \times 10^{-1}\left(\mathrm{R}^{2}=0.992\right)$ \\
$70 \mathrm{mM}$ & Scale (atomic units $)=-3.02 \times 10^{-4} \mathrm{t}+$ & Scale (atomic units $)=7.09 \times 10^{-4} \mathrm{t}-$ \\
& $2.50 \times 10^{-1}\left(\mathrm{R}^{2}=0.934\right)$ & $7.20 \times 10^{-2}\left(\mathrm{R}^{2}=0.967\right)$ \\
&
\end{tabular}

\section{References}

(1) Fogang, L. T.; Solling, T. I.; Kamal, M. S.; Sultan, A. S. Kinetics of Breaking of a Sulfobetaine Viscoelastic Surfactant Gel in the Presence of Model Oils; International Petroleum Technology Conference: Dhahran, Kingdom of Saudi Arabia, 2020.

(2) Chu, Z.; Feng, Y.; Su, X.; Han, Y. Wormlike Micelles and Solution Properties of a C22Tailed Amidosulfobetaine Surfactant. Langmuir 2010, 26, 7783-7791. 
(3) Kumar, R.; Kalur, G. C.; Ziserman, L.; Danino, D.; Raghavan, S. R. Wormlike Micelles of a C22-Tailed Zwitterionic Betaine Surfactant: From Viscoelastic Solutions to Elastic Gels. Langmuir 2007, 23, 12849-12856.

(4) Molchanov, V. S.; Philippova, O. E.; Khokhlov, A. R.; Kovalev, Y. A.; Kuklin, A. I. SelfAssembled Networks Highly Responsive to Hydrocarbons. Langmuir 2007, 23, 105-111.

(5) Zhang, Y.; An, P.; Liu, X. A "Worm"-Containing Viscoelastic Fluid Based on Single Amine Oxide Surfactant with an Unsaturated C 22 -Tail. RSC Adv 2015, 5, 19135-19144.

(6) Fogang, L. T.; Sultan, A. S.; Kamal, M. S. Understanding Viscosity Reduction of a LongTail Sulfobetaine Viscoelastic Surfactant by Organic Compounds. RSC Adv. 2018, 8, 44554463.

(7) Croce, V.; Cosgrove, T.; Dreiss, C. A.; King, S.; Maitland, G.; Hughes, T. Giant Micellar Worms under Shear: A Rheological Study Using SANS. Langmuir 2005, 21, 6762-6768.

(8) Mezger, T. G. Joe Flow 06 - Rotational Measurements. In Applied Rheology with Joe Flow on the Rheology Road; Anton Paar GmbH: Austria, 2015.

(9) Anton Paar. RheoCompass ${ }^{\mathrm{TM}}$ Software: Software Manual. Anton Paar GmbH October 2016.

(10) Fogang, L. T.; Solling, T. I.; Pedersen, J. S.; Kamal, M. S.; Sultan, A. S. Real-Time Monitoring of Oil-Induced Micellar Transitions in Viscoelastic Surfactants by Small-Angle X-Ray Scattering. J. Colloid Interface Sci. 2020, 580, 399-406. 